\title{
Pancreatitis aguda, secundaria a hiperparatiroidismo primario
}

\author{
Secondary acute pancreatitis to primary hyperparathyroidism
}

\author{
Alicia Reyes-Cerecedo,* Liliana Sayuri Tapia-Brito, ${ }^{\ddagger}$ Beatriz González-Ortiz* \\ * Servicio de Gastroenterología y Nutrición Pediátrica; ${ }^{\ddagger}$ Médico Residente en Gastroenterología y \\ Nutrición Pediátrica; Unidad Médica de Alta Especialidad, Hospital de Pediatría del Centro Médico \\ Nacional Siglo XXI, Instituto Mexicano del Seguro Social, Ciudad de México, México.
}

\begin{abstract}
RESUMEN
Introducción: Presentamos el caso de un paciente pediátrico con pancreatitis aguda secundaria a hiperparatiroidismo primario (HTPT), lo cual es una condición muy rara. Caso clínico: Masculino de nueve años y cinco meses de edad, con obesidad grave. Padecimiento de cuatro días de evolución con dolor abdominal en epigastrio que fue aumentando de intensidad progresivamente. A su ingreso, el paciente se encuentra en malas condiciones generales. Se confirmó pancreatitis por los resultados de laboratorio (lipasa 1,805 $\mathrm{U} / \mathrm{L}$, amilasa $784 \mathrm{U} / \mathrm{L}$ ) y de tomografía abdominal (colección y necrosis del páncreas). El calcio sérico estuvo persistentemente elevado. Se diagnosticó HTPT por niveles elevados de paratohormona (314.4 pg/dL) y a ultrasonido con adenoma paratiroideo. Conclusiones: El HTPT debe formar parte del escrutinio de las posibles causas de pancreatitis en niños, en particular cuando se detecta hipercalcemia.
\end{abstract}

Palabras clave: Pancreatitis aguda, hipercalcemia, hiperparatiroidismo primario, niños.

\section{INTRODUCCIÓN}

La pancreatitis aguda (PA) secundaria a hipercalcemia por hiperparatiroidismo primario es una entidad rara, por lo que se requiere de una alta sospecha para iniciar el tratamiento oportuno y evitar complicaciones en los pacientes.

Correspondencia: Alicia Reyes-Cerecedo, arcaly@hotmail.com Citar como: Reyes-Cerecedo A, Tapia-Brito LS, González-Ortiz B. Pancreatitis aguda, secundaria a hiperparatiroidismo primario. Rev Mex Pediatr. 2021; 88(2): 75-77. https://dx.doi.org/10.35366/101282

\begin{abstract}
Introduction: There are few reported cases of acute pancreatitis secondary to primary hyperparathyroidism in children. Clinical case: Nine-year-old male patient with significant obesity. His current condition of four days of evolution, with abdominal pain in the epigastrium that progressively increased in intensity. Upon admission, the patient was seriously ill. Pancreatitis was confirmed by laboratory results (lipase $1.805 \mathrm{U} / \mathrm{L}$, amylase $784 \mathrm{U} / \mathrm{L}$ ) and abdominal tomography (cyst and necrosis of the pancreas). Serum calcium was persistently elevated. Primary hyperparathyroidism was diagnosed by elevated levels of parathyroid hormone (314.4 $\mathrm{pg} / \mathrm{dL}$ ) and parathyroid adenoma identified in ultrasound. Conclusions: Primary hyperparathyroidism should be one of the possible causes of pancreatitis in children, particularly when hypercalcemia is detected.
\end{abstract}

Keywords: Acute pancreatitis, hypercalcemia, hyperparathyroidism, children.

La hipercalcemia se define como la presencia de concentraciones de calcio sérico $>10.5-11 \mathrm{mg} / \mathrm{dL},{ }^{1}$ que es secundaria al incremento de la afluencia de calcio procedente del tracto gastrointestinal o del tejido óseo al espacio extracelular, que llegan a superar la capacidad de excreción renal o por situaciones en las que existe un incremento de la reabsorción renal tubular de calcio. En el hiperparatiroidismo primario (HPTP) el origen del trastorno radica en las glándulas paratiroides: se produce una secreción excesiva y autónoma de la hormona paratiroidea a partir de la misma. En general, cuando existe hipercalcemia la 
sintomatología clínica es poco específica, lo que hace difícil su diagnóstico. A nivel digestivo puede ocurrir estreñimiento, anorexia, náusea, vómito, pirosis, úlcera péptica y pancreatitis, las cuales se encuentran en el HPTP. ${ }^{2}$

Dado que existen pocos casos publicados en la edad pediátrica, se presenta un escolar que debutó con pancreatitis aguda secundaria a HPTP.

\section{CASO CLÍNICO}

Escolar masculino de nueve años cinco meses de edad, residente de la Ciudad de México y sin antecedentes de importancia. Como el único dato previo, el paciente tenía obesidad (IMC en percentil > 99).

$\mathrm{Su}$ padecimiento de cuatro días de evolución, caracterizado únicamente por dolor abdominal leve (escala análoga visual: 3/10), el cual, $24 \mathrm{~h}$ antes de su llegada al hospital, se intensificó (10/10) y se localizó en epigastrio, siendo de tipo punzante, transfictivo, con irradiación en hemicinturón hacia región dorsal izquierda que limitaba la deambulación. El dolor se acompañó de vómito de contenido biliar en varias ocasiones. No se identificó fiebre.

A su ingreso en el servicio de Urgencias, el paciente se recibió en malas condiciones generales, con datos de dificultad respiratoria, polipnea, saturación de oxígeno de $70 \%$ y Glasgow 8/15, por lo que se inició manejo avanzado de la vía aérea y ventilación mecánica.

Se indicó ayuno, soluciones parenterales, manejo del dolor con medicamentos no especificados y colocación de sonda nasogástrica (SNG), obteniendo drenaje de contenido gastrobiliar.

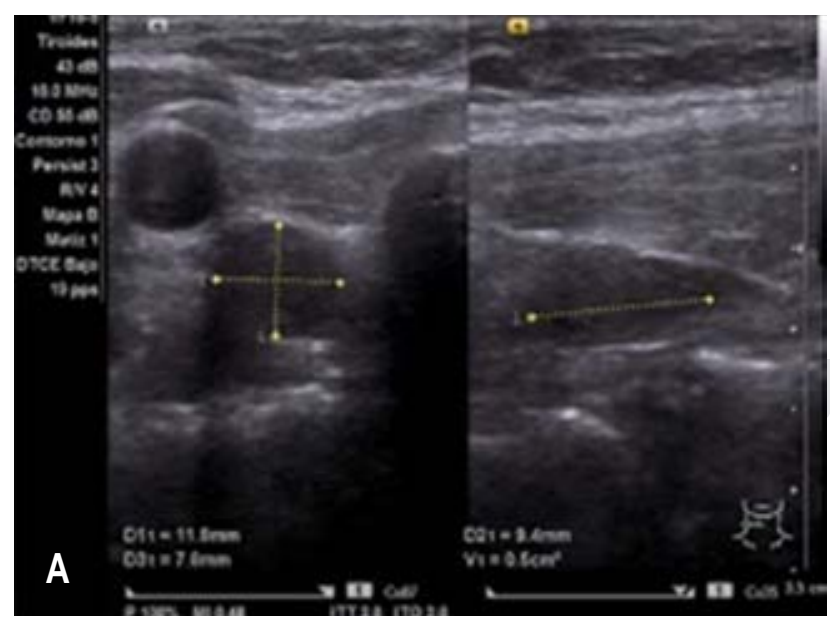

En los estudios de laboratorio se determinó acidosis respiratoria descompensada, hiperglucemia, hipercalcemia, elevación de azoados y disminución de depuración de creatinina (TFG $48.1 \mathrm{~mL} / \mathrm{min} / 1.73$ $\mathrm{m}^{2}$ ), además de elevación sérica de lipasa 1,805 U/L y amilasa 784 U/L. Dada la gravedad del paciente, se ingresó a la Unidad de Cuidados Intensivos Pediátricos (UCIP) con diagnóstico de pancreatitis aguda grave.

A fin de determinar la etiología de la pancreatitis, mediante estudios de imagen se buscaron y descartaron causas obstructivas, biliares y anatómicas.

Un aspecto que llamó la atención fue la persistencia de elevación de calcio sérico (hasta 15.0 $\mathrm{mg} / \mathrm{dL})$ y calcio iónico $(2.0 \mathrm{mmol} / \mathrm{L})$, por lo que se iniciaron medidas antihipercalcemia con manejo de líquidos a $2,500 \mathrm{~mL} / \mathrm{m}^{2} / \mathrm{SC}$ y diurético de asa durante $48 \mathrm{~h}$. Ante la falta de respuesta, se decidió administrar ácido zoledrónico, como quelante de calcio. Por lo anterior, se solicitó determinación de paratohormona en sangre siendo el valor de 314.4 $\mathrm{pg} / \mathrm{dL}$ (normal 10-55 pg/dL). Además, el fósforo sérico fue de $4.26 \mathrm{mg} / \mathrm{dL}$, se detectó hipercalciuria y niveles séricos de vitamina $\mathrm{D}$ en $6.8 \mathrm{ng} / \mathrm{dL}$ (normal de 30-40 n/dL).

Mediante ultrasonido (USG) tiroideo se observó una imagen nodular hipoecogénica de $11.8 \times 9.4 \times 7.8$ $\mathrm{mm}$, volumen de $0.5 \mathrm{~cm}^{3}$, localizada posterior al lóbulo tiroideo derecho, con vascularidad interna, sugestiva de adenoma paratiroideo (Figura 1). Con esta información más los datos de laboratorio se llegó al diagnóstico de pancreatitis aguda grave secundaria a hipercalcemia por HPTP.

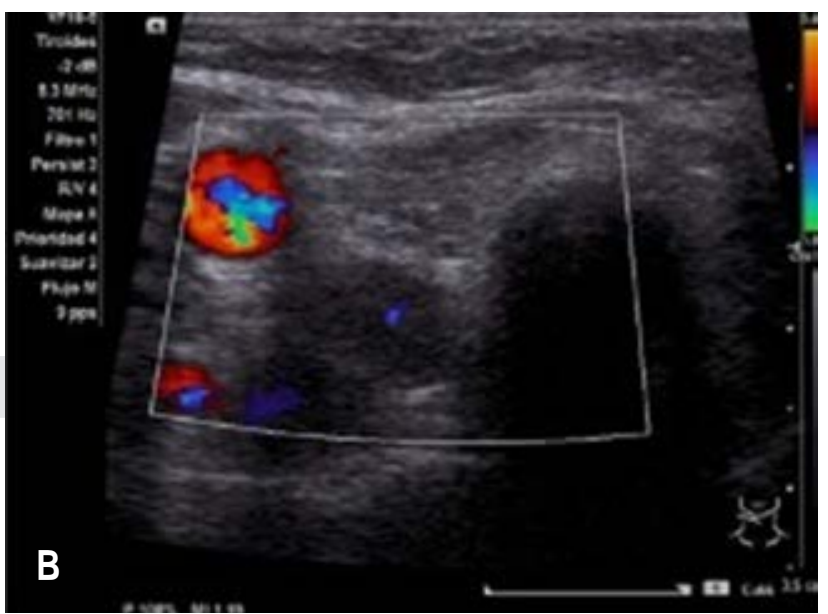

Figura 1: A) Ultrasonido tiroideo, B) adenoma paratiroideo de $1.7 \times 1.0 \mathrm{~cm}$ y $1.1 \mathrm{~g}$. 


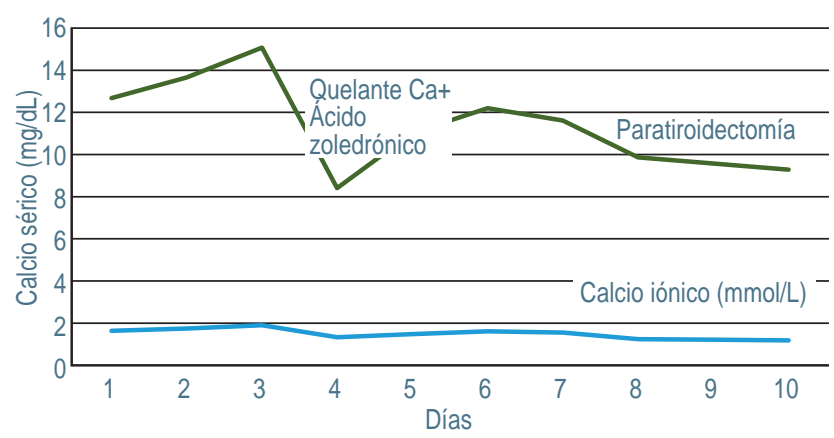

Figura 2: Evolución de los niveles de calcio sérico e iónico.

El paciente permaneció en la UCIP durante 10 días hasta la resolución del estado de gravedad. En cuanto se obtuvo mejoría, se decidió realizar paratiroidectomía de las glándulas paratiroides superior e inferior derecha. Tras el evento quirúrgico, se obtuvo normalización de los niveles de calcio y de los niveles de paratohormona. El reporte histopatológico fue de un adenoma paratiroideo. En la Figura 2 se muestra la evolución del calcio sérico y iónico durante 10 días.

\section{DISCUSIÓN}

La hipercalcemia secundaria a HPTP es una causa poco frecuente de pancreatitis aguda. Se cree que la pérdida del mecanismo regulador de paratohormona y la hipercalcemia favorecen el depósito de calcio en los conductos pancreáticos y la activación de enzimas pancreáticas. $^{3}$

Los pacientes con HPTP e hipercalcemia tienen 10 veces mayor riesgo que la población general de padecer pancreatitis aguda; ${ }^{4}$ lo habitual es que la pancreatitis aguda se desarrolle en un paciente ya conocido con hiperparatiroidismo. El caso que aquí presentamos es particular, ya que hay muy pocos casos de ambas condiciones en pacientes pediátricos. ${ }^{5-7}$

Aunque no se ha podido establecer una base fisiopatológica, existen algunas hipótesis que tratan de explicar esta relación. Kelly et al. demostraron, mediante un modelo experimental, que la calcemia elevada provoca un incremento de la concentración de calcio en el jugo pancreático, lo cual favorece la transformación de tripsinógeno en tripsina activa. ${ }^{3}$ Más recientemente se ha comprobado que la elevación de calcio en el citosol desencadena pancreatitis. ${ }^{4,8} \mathrm{Se}$ cree que la hipercalcemia promovería la activación de las enzimas pancreáticas por medio de las hidrolasas ácidas lisosomales. También se ha implicado al gen CASR (gen de los receptores de calcio), que participa en la regulación de la permeabilidad de la membrana lisosomal, en la homeostasis del calcio intracelular y en el control de la activación de la tripsina, así parece ser que las mutaciones en los genes facilita el desarrollo de pancreatitis..$^{8,9}$

Se ha reportado en la literatura que hasta $80 \%$ de los casos el HPTP se origina por un adenoma paratiroideo. ${ }^{10} \mathrm{El}$ paciente que reportamos no presentaba a la palpación algún nódulo en la región tiroidea, por lo que se realizó búsqueda intencionada por ultrasonido, encontrándose el nódulo en el polo inferior del lóbulo derecho de la glándula paratiroides (Figura 1).

\section{AGRADECIMIENTOS}

Al Servicio de Radiología de la UMAE Hospital de Pediatría.

\section{REFERENCIAS}

1. Carroll MF, Schade DS. A practical approach to hypercalcemia. Am Fam Physician. 2003; 67: 1959-7966.

2. Eastell R, Arnold A, Brandi ML, Brown EM, D’Amour P, Hanley DA et al. Diagnosis of asymptomatic primary hyperparathyroidism: proceedings of the third international workshop. J Clin Endocrinol Metab. 2009; 94(2): 340-350.

3. Kelly TR. Relationship of hyperparathyroidism to pancreatitis. Arch Surg. 1968; 97(2): 267-274.

4. Chowdhury SD, Kurien RT, Pal S, Jeyaraj V, Joseph AJ, Dutta AK et al. Acute pancreatitis and hyperparathyroidism: a case series. Indian J Gastroenterol. 2014; 33(2): 175-177.

5. Roizen J, Levine MA. Primary hyperparathyroidism in children and adolescents. J Chin Med Assoc. 2012;75(9):425-34.

6. Tsuboi K, Takamura M, Sato Y, Yokoyama H, Takeuchi M, Igarashi Mel et al. Severe acute pancreatitis as an initial manifestation of primary hyperparathyroid adenoma in a pediatric patient. Pancreas. 2007; 35(1):100-1.

7. Gupta AK, Madnani M, Mistry J, Soni H, Shah A, Patel KS, et al. Primary hyperparathyroidism with pancreatitis: experience of management in 5 patients with review of literature. Indian $J$ Gastroenterol. 2014;33(5):484-6.

8. Bai HX, Giefer M, Patel M, Orabi AI, Husain SZ. The association of primary hyperparathyroidism with pancreatitis. J Clin Gastroenterol. 2012; 46(8): 656-661.

9. Abu-El-Haija M, Kumar S, Quiros JA, Balakrishnan K, Barth B, Bitton $S$ et al. Management of acute pancreatitis in the pediatric population: a clinical report from the North American Society for Pediatric Gastroenterology, Hepatology and Nutrition Pancreas Committee. J Pediatr Gastroenterol Nutr. 2018; 66(1): 159-176.

10. Shimonov M, Leibou L, Shechter P, Judich A. Pancreatitis due to hypercalcemia in a young adult. Isr Med Assoc J. 2012; 14(4): 267-268.

Conflicto de intereses: Las autoras declaran que no tienen. 